\title{
Children's performance on set-inclusion and linear-ordering relationships
}

\author{
STEPHEN E. NEWSTEAD \\ Plymouth Polytechnic, Plymouth, England \\ STEPHANIE KEEBLE \\ MRC Cognitive Development Unit, London, England \\ and \\ KENNETH I. MANKTELOW \\ Sunderland Polytechnic, Sunderland, England
}

\begin{abstract}
Children between the ages of 6 and 16 years were presented with set-inclusion and linearordering relationships embedded in text. On the set-inclusion task, children of all age groups performed similarly to adult subjects; that is, there was an interaction between truth and distance. Hence, children, like adults, treated the relationship as if it were convertible and nontransitive, both of which can be regarded as logical errors. It was suggested that this pattern of results may result from a basic response bias. On the linear-ordering task, young children had great difficulty both in remembering the material and in drawing the appropriate inferences. It was concluded that children may acquire transitivity later on verbally presented tasks than on visually presented ones.
\end{abstract}

In the reasoning literature, there is a paradox in the performance of adult subjects on linear-ordering and setinclusion tasks. People have no difficulty with linearordering tasks, correctly drawing the permitted transitive inferences. With set inclusions, however, people tend not to draw transitive inferences, and performance in general is poor. In this paper, we try to throw light on this paradox by examining developmental trends in performance on the two tasks.

A study by Potts (1974) is a fairly typical one using linear orderings. Subjects were presented with a verbal passage containing the information $\mathrm{A}>\mathrm{B}>\mathrm{C}>\mathrm{D}$, where $>$ indicates a relationship such as "better than" and the letters represent entities. Subjects were then presented with true-false test items, some of which involved transitive inferences (e.g., A > D). Subjects had no difficulty with such test items, and, indeed, performance actually tended to be superior on inferential items; hence, subjects could draw transitive inferences with consummate ease.

A typical set-inclusion task is that of Griggs (1976). Subjects were presented with passages containing the information that All As are Bs, All Bs are Cs, All Cs are $D s$. When presented with true-false test items, many subjects did not draw the permitted transitive inferences (e.g., All As are Ds). In fact, the greater the inferential distance

Part of this research was carried out while the first author was on sabbatical leave at the University of Florida. The authors would like to thank Rich Griggs for his advice and comments and Julia Billington for her help in running Experiment 3. The first author's mailing address is: Department of Psychology, Plymouth Polytechnic, Drake Circus, Plymouth PL4 8AA, England. between the terms in the test item, the less likely subjects were to draw the inference-the exact reverse of the distance effect found with linear orderings. In addition, to errors of transitivity, subjects also tended to make conversion errors with set inclusions. Thus, subjects believed the converted versions of the premise information (e.g., All Bs are As) to be true. In fact, one can deduce neither the truth nor the falsity of converted items, but, under the instructions given in such tasks, they should be deemed false. This is because the instructions ask subjects to indicate as true only test items that were in the original premises or that logically followed from them, and otherwise to call items false.

Overall, the pattern of performance on set-inclusion tasks can by typified as a truth $\times$ distance interaction. For true items, accuracy is good on adjacent items (the original premises) but declines over inferential distance. For false items, performance is poor on adjacent items (i.e., converted premises) but improves over inferential distance, since subjects are more likely to call such items false. This pattern of responding has proved to be remarkably robust over different populations, different experimental paradigms, and different types of test materials.

Several different explanations of why subjects should respond in this way have been put forward, but none is entirely satisfactory. Potts (1978) suggested that set inclusions are incorrectly treated as similarity relations, which are nontransitive and convertible; however, he provided no persuasive argument as to why set inclusions should be so misinterpreted. Mynatt and Smith (1979) claimed that the errors are due to inappropriate schema cuing; they claimed that subjects can process inclusions 
appropriately, for example, if these involve geographical or topographical inclusions (e.g., $A$ is in $B$ ). However, the normal set inclusion using the quantifier all leads to inappropriate processing, although the authors gave no sound reason why this should occur. In fact, a possible explanation may be found in a third theoretical approach to set inclusions, that of Newstead and Griggs (1983, 1984). Their general argument is that errors may be due to subjects' misinterpreting the meaning of all or, more precisely, to subjects' interpreting all in terms of normal linguistic usage rather than according to the dictates of logic. However, the experimental work carried out by these authors suggested that, although such misinterpretations could explain conversion errors, they could not explain errors of transitivity. Hence, there is, at the present moment, no completely satisfactory account of set-inclusion performance.

A primary aim of the present reseach was to throw further light on set inclusions. The approach adopted was to examine developmental trends in performance to find out at what age the adult pattern of responding first occurs. To date, there appear to have been no studies of children's performance on this task.

The present study also investigated children's performance on linear orderings, in part to provide a comparison with set inclusions. In sharp contrast to the case of set inclusions, there have been extensive developmental studies in this area. Early researchers such as Burt (1919) and Piaget (1921) used three-term linear orderings, or linear syllogisms, such as John is taller than Dick; Dick is taller than Alan; Who is the tallest? They claimed that the ability to draw transitive inferences did not emerge until the concrete operations stage or later. The main challenge to this position came from Bryant and Trabasso (1971), who claimed that, providing that precautions were taken to ensure that the premises were remembered, even children of 4 or 5 years of age could draw the correct inferences. Although there have been dissenters (e.g., Breslow, 1981), in general, Bryant and Trabasso's conclusions have been widely accepted.

However, it is interesting to note that the recent studies demonstrating transitivity in young children have used mostly nonverbal presentation (Bryant \& Trabasso, 1971, used five colored sticks of different lengths). The early work by Burt (1919) and Piaget (1921), which failed to show transitivity, used verbally presented linear syllogisms. However, two unpublished studies using verbal tasks appear to have demonstrated transitivity in young children. Stetson (1974) gave children of 7, 9, and 11 years of age repeated presentations of stories involving transitive relationships until the children could remember them. The results suggested that children from all age groups could draw the appropriate inferences. Similarly, Bryant and Perkins (cited in Bryant, 1973) presented 5year-old children with a story containing a transitive chain and found that they could draw the inferences. Clearly, further research is required on verbal presentation of linear orderings, and this is a further aim of the present study.
The research just mentioned would lead to the prediction that even young children will demonstrate transitivity. If this is so, the question arises as to whether these children will show the distance effect in which performance is actually better on inferential items.

\section{EXPERIMENTS 1, 2, AND 3}

Although these experiments were run individually, and there were methodological differences among them, the general procedures were similar, and so were the patterns of results. For this reason, the experiments are described together, with any differences among them being mentioned at the appropriate points.

\section{Method}

Subjects. There were 96 subjects in each experiment, making a total of 288 children, $\mathbf{4 8}$ from each of six age groups. The youngest age group, which will be referred to as the 6-year-olds, comprised children of 6.37.4 years of age. The 8-year-olds were 8.3-9.4 years of age; the 10year-olds, 10.3-11.4 years; the 12-year-olds, 12.1-13.4 years; the 14year-olds, 13.5-15.4 years; and the 16-year-olds, 16.2-18.1 years. The age ranges given are for the classes from which the children were drawn; sampling was random and anonymous. Half the children from each age group were male, and half were female.

In Experiments 1 and 3, the children were drawn from nonselective schools in Tavistock and Ivybridge (both in Devon, England), respectively. In Experiment 2, all the children were drawn from the P. K. Yonge Laboratory School, Gainesville, Florida, U.S.A.

Materials. There were two set-inclusion passages, one involving sweets, the other toy bricks. The "sweets" passage was:

Some children were deciding which sweets to buy. All the round sweets were chocolates. All the chocolates were large. All the large sweets were hard. They all went to the shop and bought what they wanted.

The two passages using linear orders also involved sweets or toy bricks linked by the relationship "better than." The passages were "Americanized" for Experiment 2: "sweets" became "candies," and "toy bricks" became "toy blocks." In Experiment 1, the premises were presented in serial order (i.e., A-B, B-C, C-D); in Experiments 2 and 3 , however, half the subjects received the premises in nonserial order (B-C, C-D, A-B).

Procedure. Each subject was given one linear-ordering passage and one set-inclusion passage. Half the subjects received the linear-ordering passage first, and half the set-inclusion passage first. The material used (sweets or bricks) was always different in the two passages. The subjects were tested individually. They were told that the experimenter would read a passage to them, after which they would be asked to answer a number of questions about the passage. In Experiments 1 and 2, the children were allowed as many repetitions of the passage as they liked. On average, they requested 2.2 presentations, and there were no age differences in the number requested. Since the younger children may have asked for relatively few repetitions because of their poor metamemory, all subjects were presented with the passage precisely three times in Experiment 3. After the passage had been presented, the subjects were given 12 oral questions concerning the relationships between pairs of items in the passage, to which the children had to respond simply "yes" or "no." Three of these questions concerned pairs already presented in the premises (e.g., "Were all the round sweets chocolates?" for the set-inclusion passage given in the Materials sections). Three questions involved valid remote inferences (e.g., "Were all the round sweets large?'"). The remaining questions involved the reversals of the three valid premises and three valid inferences. The questions were presented in random order. The second passage was presented immediately after the questioning on the first passage had finished. 


\section{Results}

The results consisted of the proportion of correct answers given. These will be considered separately for the two relationships used.

Set inclusions. The data were submitted to a three-way analysis of variance in which the factors were age $(6,8$, $10,12,14$, or 16 years old), distance (adjacent or remote), and truth (true or false). True items are those that simply repeat the premises or present valid transitive inferences. False items are defined as the reversals of these items; strictly speaking, such items are indeterminate, but the present terminology is used for consistency with adult studies. Distance refers to whether the terms are adjacent (i.e., the original premises and their reversals) or remote (i.e., inferential items and their reversals). There was a main effect of truth $[F(1,282)=89.34, p<.001]$, a main effect of distance $[\mathrm{F}(1,282)=8.36, \mathrm{p}<.01]$, and an interaction between truth and distance $[\mathrm{F}(1,282)=$ $143.59, \mathrm{p}<.001]$. Although there was a significant interaction between age, truth, and distance $[F(5,282)=$ $2.68, \mathrm{p}<.05$ ], the interaction was highly similar for all age groups. With true items, accuracy declined over inferential distance, from .8 on adjacents to .55 on remotes. However, with false items, performance improved from .29 on adjacents to .47 on remotes. This interaction is remarkably similar to that found with adults.

Since the truth $\times$ distance interaction is marked in all the age groups studied, we wondered whether even younger children children would behave in a similar way. To this end, a group of 4-year-old children, drawn from Ivybridge preschool playgroup, Devon, were run with a procedure identical to that used with other subjects in Experiment 3 . There was no truth $\times$ distance interaction on the set inclusions $(F<1)$, but, instead, a massive effect of truth $[\mathrm{F}(1,30)=334.5, \mathrm{p}<.001]$. These children were responding "yes" to nearly all the test items, regardless of their truth value. (Interestingly, this pattern of responses was duplicated on the linear-ordering task.) Thus, these 4-year-olds showed a pattern of responding very different from that of children from older groups.

Linear orderings. The data were submitted to the same three-way analysis of variance that was used on set inclusions. There was a main effect of truth, with true responses producing more correct responses $(72 \%)$ than false ones $(68 \%)[\mathrm{F}(1,282)=4.95, \mathrm{p}<.05]$. There was a main effect of age $[\mathrm{F}(5,282)=6.33, \mathrm{p}<.001]$ and an interaction between age and distance $[F(5,282)=2.72$, $\mathrm{p}<.05$ ], which is plotted in Table 1 . There was no significant difference between adjacents and remotes for 6and 8 -year-olds, with performance being marginally better on adjacents. For 10-year-olds, performance was significantly better on remotes $[t(47)=3.22, p<.01]$. For 12 - and 16-year-olds, performance was also better on remotes, and this difference approached significance with the 16-year-olds [t(47) $=1.51, \mathrm{p}<.1]$. However, there appears to be no difference between adjacents and remotes in the data for the 14-year-olds. Although these results are not clear-cut, there is evidence that any distance effects in this task do not appear before the age of 10 years.
Table 1

Proportion Correct on Linear Orderings as a Function of Age and Distance

\begin{tabular}{lllllll}
\hline & \multicolumn{6}{c}{ Age } \\
\cline { 2 - 7 } & 6 & 8 & 10 & 12 & 14 & 16 \\
\hline Adjacents & .57 & .67 & .67 & .73 & .77 & .72 \\
Remotes & .55 & .63 & .78 & .77 & .76 & .77 \\
\hline
\end{tabular}

In order to determine whether children of each age group were performing at above chance level (.5) on the inferences (i.e., the remote items in Table 1), one-tailed sign tests were carried out. With children 8 years old and older, performance was significantly better than chance $(p<.01)$. However, the performance of the 6-year-olds did not differ significantly from chance $(\mathrm{p}=.42)$. Thus, although there was good evidence that the older children could draw the appropriate transitive inferences, there was no evidence that the younger children could.

However, this analysis fails to take into consideration whether the children could remember the premises. In order to provide a check on memory, the data were reanalyzed for only those children who had responded correctly to the premises. Once again, children 8 years old and older performed at better than chance level throughout. The 6year-olds, however, could not be analyzed in this way, since there were so few subjects ( 4 of 48 ) who achieved the memory criterion. Although it is clear that 6-year-olds do not draw the permitted inferences, it is impossible to say whether this is due to an inability to handle transitivity or to an inability to remember the premises.

As a further check on the effects of memory, an additional experiment was carried out with a five-term linear ordering in which children 6,8 , and 10 years old were trained to a criterion of two correct repetitions. In fact, it proved impossible to train more than a handful of the 6-year-olds to such a criterion, and the attempt was abandoned; this serves to confirm the difficulty such young children have in remembering verbally presented linear orderings. It was possible to train the 8- and 10-year-olds to criterion, but a rather surprising finding emerged. Despite the training, these children did not perform impressively on inferences (approximately $70 \%$ accuracy) and, more surprisingly, did not perform well on the premises (again, approximately $70 \%$ accuracy). It would appear that verbatim repetition of a passage is no guarantee that individual statements will be responded to correctly.

\section{DISCUSSION}

One of the main questions raised in the introduction concerned the age at which children would first show the truth $\times$ distance interaction on set-inclusion tasks. It is evident that the interaction emerges very early, being clearly present in 6-year-old children. However, a followup experiment on even younger children (4-year-olds) produced no evidence of an interaction; instead, children responded "yes" to all items, probably because of a response bias.

Since the interactions are so similar, it seems highly probable that the same processes underlie the performance of both adults and children. The explanations given of the adult results-whether in terms of inappropriate schema cuing, misinterpretation of the quantifiers, or treat- 
ing the relationship as one of similarity-all involve rather subtle and complicated processes. The fact that very young children produce similar results suggests that the explanation may actually be more simple. One possibility, alluded to by Newstead and Griggs (1984), is that any relationship that is not fully understood may be assumed, by default, to be convertible and nontransitive. Alternatively, one could give an associationistic account of the results. Adjacent terms may be associated with each other through contiguity, since they occur in the same premise; as a consequence, any test item containing adjacent terms, whether converted or not, might be judged to be true. With remote items, the association will be less strong-hence the increased tendency to respond false. Such basic explanations of the interaction certainly deserve further investigation.

Another principal question raised in the introduction concerned children's ability to draw transitive inferences from a verbally presented linear ordering. Although performance on inferences was surprisingly low overall, there was evidence that children 8 years old and older could draw the appropriate inferences. The data from the 6-year-olds provided no evidence of ability to draw transitive inferences, but this may be attributable to the difficulty they had in remembering the premises. Furthermore, there was surprisingly little evidence for the existence of the distance effects that have previously been found in both adult and child studies. There were no such effects in 6- and 8-year-old children, and they were far from dramatic in the older children. It appears that transitive inferences and distance effects may emerge later and be less marked on verbal, as opposed to nonverbal, tasks. The fact that the unpublished study by Bryant and Perkins (cited in Bryant, 1973) did find such effects with a verbal task may indicate that there is variability between different verbal tasks.

\section{REFERENCES}

BresLow, L. (1981). Re-evaluation of the literature on the development of transitive inferences. Psychological Bulletin, 89, 325-351.

Bryant, P. E. (1973). What the young child has to learn about logic. In R. A. Hinde \& J. S. Hinde, (Eds.), Constraints on learning. London: Academic Press.

Bryant, P. E., \& Trabasso, T. (1971). Transitive inferences and memory in young children. Nature, 232, 456-458.

BuRT, C. (1919). The development of reasoning in school children. Journal of Experimental Pedagogy, 5, 68-77.

GRIGGS, R. A. (1976). Logical processing of set inclusion relations in meaningful text. Memory \& Cognition, 4, 730-740.

Mynatt, B. T., \& Smith, K. H. (1979). Processing of text containing artificial inclusion relations. Memory \& Cognition, 7, 390-400.

Newstead, S. E., \& Griggs, R. A. (1983). Drawing inferences from quantified statements: A study of the square of opposition. Journal of Verbal Learning and Verbal Behavior, 22, 535-546.

Newstead, S. E., \& Griggs, R. A. (1984). Fuzzy quantifiers as an explanation of set inclusion performance. Psychological Research, 46, 379-388.

Piaget, J. (1921). Une forme verbale de la comparaison chez l'enfant. Archives de Psychologie, 18, 141-172.

PoTTs, G. R. (1974). Storing and retrieving information about ordered relationships. Journal of Experimental Psychology, 103, 431-439.

STETSON, P. C. (1974). Verbal transitivity in children. Unpublished doctoral dissertation, University of Delaware.

(Revision received for publication November 23, 1984.) 